\title{
Periodic parabolic problems with concave and convex nonlinearities*
}

\author{
T. GODOY and U. KAUFMANN \\ Facultad de Matemática, Astronomía y Física \\ Universidad Nacional de Córdoba \\ Ciudad Universitaria \\ 5000 Córdoba, Argentina \\ e-mail: godoy@mate.uncor.edu \\ e-mail: kaufmann@mate.uncor.edu
}

\begin{abstract}
Let $\Omega \subset \mathbb{R}^{N}$ be a smooth bounded domain, let $a, b$ be two functions that are possibly discontinuous and unbounded with $a \geq 0$ in $\Omega \times \mathbb{R}$ and $b>0$ in a set of positive measure and let $0<p<1<q$. We prove that there exists some $0<\Lambda<\infty$ such that the nonlinear Dirichlet periodic parabolic problem $L u=\lambda a(x, t) u^{p}+b(x, t) u^{q}$ in $\Omega \times \mathbb{R}$ has a positive solution for all $0<\lambda<\Lambda$ and that there is no positive solution if $\lambda>\Lambda$. In some cases we also show the existence of a minimal solution for all $0<\lambda<\Lambda$ and that the solution $u_{\lambda}$ can be chosen such that $\lambda \rightarrow u_{\lambda}$ is differentiable and increasing. We also give some upper and lower estimates for such a $\Lambda$. All results remain true for the analogous elliptic problems.
\end{abstract}

2000 Mathematics Subject Classification: 35K20, 35P05, 35B10

Key words: parabolic, periodic, concave, convex

\section{Introduction}

Let $\Omega$ be a $C^{2+\theta}$ bounded domain in $\mathbb{R}^{N}, N \geq 2, \theta \in(0,1)$. For $T>0$ and $1 \leq p \leq \infty$, let $L_{T}^{p}$ be the Banach space of $T$-periodic functions $f$ on $\Omega \times \mathbb{R}$ (i.e. satisfying $f(x, t)=f(x, t+T)$ a.e. $(x, t) \in \Omega \times \mathbb{R})$ such that $f_{\mid \Omega \times(0, T)} \in$ $L^{p}(\Omega \times(0, T))$, equipped with the norm $\|f\|_{L_{T}^{p}}:=\left\|f_{\mid \Omega \times(0, T)}\right\|_{L^{p}(\Omega \times(0, T))}$. Let $C_{T}$ be the space of continuous and $T$-periodic functions on $\bar{\Omega} \times \mathbb{R}$ provided with the $L^{\infty}$ norm, and let $C_{T}^{1+\theta,(1+\theta) / 2}$ be the space of $T$-periodic functions belonging to $C^{1+\theta,(1+\theta) / 2}(\bar{\Omega} \times \mathbb{R})$.

*Partially supported by CONICET, Secyt-UNC, ANPCYT and Agencia Cordoba Ciencia 
Let $\left\{a_{i j}\right\},\left\{b_{j}\right\}, 1 \leq i, j \leq N$, be two families of $T$-periodic functions satisfying $a_{i j} \in C^{0,1}(\bar{\Omega} \times \mathbb{R}), a_{i j}=a_{j i}$ and $b_{j} \in L_{T}^{\infty}$, and assume that

$$
\sum a_{i j}(x, t) \xi_{i} \xi_{j} \geq \alpha_{0}|\xi|^{2}
$$

for some $\alpha_{0}>0$ and all $(x, t) \in \Omega \times \mathbb{R}, \xi \in \mathbb{R}^{N}$. Let $A$ be the $N \times N$ matrix whose $i, j$ entry is $a_{i j}$, let $\bar{b}=\left(b_{1}, \ldots, b_{N}\right)$, let $0 \leq c_{0} \in L_{T}^{r}, r>N+2$, and let $L$ be the parabolic operator given by

$$
L u=u_{t}-\operatorname{div}(A \nabla u)+\langle\bar{b}, \nabla u\rangle+c_{0} u .
$$

Let $W=\left\{u \in L^{2}\left((0, T), H_{0}^{1}(\Omega)\right): u_{t} \in L^{2}\left((0, T), H^{-1}(\Omega)\right)\right\}$. For a given $f \in$ $L_{T}^{2}$, we will say that $u$ is a (weak) solution of the periodic problem

$$
\begin{cases}L u=f & \text { in } \Omega \times \mathbb{R} \\ u=0 & \text { on } \partial \Omega \times \mathbb{R} \\ u T \text {-periodic } & \end{cases}
$$

if $u$ is $T$-periodic, $u_{\mid \Omega \times(0, T)} \in W$ and

$$
\int_{\Omega \times(0, T)}\left[-u \frac{\partial h}{\partial t}+\langle A \nabla u, \nabla h\rangle+\langle\bar{b}, \nabla u\rangle h+c_{0} u h\right]=\int_{\Omega \times(0, T)} f h
$$

for all $h \in C_{c}^{\infty}(\Omega \times(0, T))$. For $u \in W$, the inequality $L u \geq f($ resp. $\leq)$ in $\Omega \times \mathbb{R}$, $u=0$ in $\partial \Omega \times \mathbb{R}$ will be understood in the analogous weak sense.

For $1 \leq r \leq \infty$ let $W_{r}^{2,1}\left(\Omega \times\left(t_{0}, t_{1}\right)\right)$ be the Sobolev space of the functions $u \in L^{r}\left(\Omega \times\left(t_{0}, t_{1}\right)\right), u=u(x, t), x=\left(x_{1}, \ldots, . x_{N}\right)$ such that $u_{t}, u_{x_{j}}$ and $u_{x_{i} x_{j}}$ belong to $L^{r}\left(\Omega \times\left(t_{0}, t_{1}\right)\right)$ for $1 \leq i, j \leq N$, and let $W_{r, T}^{2,1}$ be the space of $T$-periodic functions such that $u_{\mid \Omega \times(0, T)} \in W_{r}^{2,1}(\Omega \times(0, T))$. For $f \in L_{T}^{r}, r>1$, we say that $u$ is a strong solution of $(1.1)$ if $u \in W_{r, T}^{2,1}(\Omega \times \mathbb{R})$ and the equation holds a.e. in the pointwise sense.

The existence of positive solutions for periodic parabolic problems of the form

$$
\begin{cases}L u=g(x, t, u) & \text { in } \Omega \times \mathbb{R} \\ u=0 & \text { on } \partial \Omega \times \mathbb{R} \\ u T \text {-periodic } & \end{cases}
$$

has been widely studied (see e.g. [13] and the references therein). For applications we refer to [13], [3]. In [8] and [11], bifurcation of positive solutions for (1.2) was proved assuming that $\xi \rightarrow g(x, t, \xi) / \xi$ is nonincreasing in $(0, \infty)$ and that $g_{\xi}(x, t, 0)$ belongs to $L_{T}^{r}$ for some $r>(N+2) / 2$. On the other hand, in [9], existence results of positive solutions for (1.2) were given without monotonicity conditions on $g$ and allowing $g_{\xi}(x, t, 0)=+\infty$, but assuming that $\sup _{0<\xi \leq \sigma}$ 
$(g(x, t, \sigma) / \sigma)$ belongs to $L_{T}^{r}$ for some $r>(N+2) / 2$. However, in many cases of interest neither of the above conditions hold. A typical example of this situation is the problem

$$
\begin{cases}L u=\lambda a(x, t) u^{p}+b(x, t) u^{q} & \text { in } \Omega \times \mathbb{R} \\ u=0 & \text { on } \partial \Omega \times \mathbb{R} \\ u T \text {-periodic } & \end{cases}
$$

where $\lambda>0$ is a real parameter, $0<p<1<q$, and $a, b$ are two $T$-periodic functions such that $a \geq 0$ in $\Omega \times \mathbb{R}$ and $b>0$ in a subset of $\Omega \times \mathbb{R}$ of positive measure.

Our aim in this paper is to study (1.3), where $a, b$ belong to $L_{T}^{r}$ with $r>$ $N+2$. Concerning this problem, we will prove that there exists some $0<\Lambda<\infty$ such that (1.3) has a positive solution for all $0<\lambda<\Lambda$ and that there is no positive solution if $\lambda>\Lambda$. These results will follow from a sub and supersolution approach combined with some facts about linear problems with weight and some related semilinear problems (see Theorem 3.1 (i)). Moreover, when $b \geq 0$ we will show the existence of a minimal solution for all $0<\lambda<\Lambda$, and in some cases we will also prove by means of the implicit function theorem the existence of solutions $u_{\lambda}$ such that $\lambda \rightarrow u_{\lambda}$ is differentiable and increasing (see Theorem 3.1 (ii) and Theorem 3.3 respectively, and also Remark 3.4). Finally, some upper and lower estimates for $\Lambda$ are given (see Theorem 3.5 and also Remark 3.6).

To avoid unnecessary complexity, we restrict ourselves to (1.3), but one can see that most of the results are still valid for increasing nonlinearities that behave like $u^{p}$ and $u^{q}$ near the origin and infinity. We mention also that as a consequence of our proofs all results remain true for the analogous elliptic problem.

In order to relate our results to others in the literature, let us mention that existence and multiplicity of positive solutions for the corresponding elliptic problem to (1.3) was considered in detail by Ambrosetti-Brezis-Cerami in the well-known paper [1] for $L=-\Delta$ and $a=b \equiv 1$, and a generalization of these results was given in [6] (see also the references therein) also for $L=-\Delta$. On the other hand, for a non-selfadjoint operator, some existence results have been proved recently for $0 \leq a, b \in C^{1}(\Omega)$ and allowing these functions to have a singularity in the boundary in [12], Section 3, and also in [4] assuming that $a \equiv 1$ and $b \in C^{\alpha}(\bar{\Omega}), \alpha \in(0,1)$. In the parabolic case, when $b \leq 0$ (logistic-type equation), existence of positive periodic solutions was shown in [9], but no results seem to be known for (1.3) when $b>0$ in a set of positive measure. We refer to [15], [14], [9], [10] and its references for similar periodic parabolic problems.

Let us conclude this introduction with some comments on the matter of multiplicity of positive solutions for (1.3). In both [1] and [6], existence of at least two (positive) solutions for (1.3) is proved under some growth restriction on $q$ (namely, $q \leq(N+2) /(N-2))$ using variational arguments, which of course are not eligible in our case. On the other hand, also under some restrictions on $b$ and $q$, in [4] a second (positive) solution is found in a different way assuming that $L$ is selfadjoint and making use of some spectral theory with singular potential 
(see [12]) which is not available for the periodic parabolic problem. We believe that at least some of the above mentioned multiplicity results should still be true in the parabolic case, but we are not able to give a proof. What indeed can be proved for our problem (with a different proof than the one given in [1]) is the following uniqueness result: there exists $c>0$ such that (1.3) has at most one positive solution $u$ with $\|u\|_{\infty} \leq c$ (see Remark 3.2 below).

\section{Preliminaries and auxiliary results}

We start collecting some known facts about periodic parabolic problems with weight.

Remark 2.1 (i) Let $m \in L_{T}^{r}, r>(N+2) / 2$, and let

$$
P(m):=\int_{0}^{T} \operatorname{esssup}_{x \in \Omega} m(x, t) d t .
$$

Then $P(m)>0$ is necessary and sufficient for the existence of a (unique) positive principal eigenvalue $\lambda_{1}(m)$ for the problem

$$
\begin{cases}L u=\lambda m u & \text { in } \Omega \times \mathbb{R} \\ u=0 & \text { on } \partial \Omega \times \mathbb{R} \\ u T \text {-periodic } & \end{cases}
$$

It also holds that $m \rightarrow \lambda_{1}(m)$ is a continuous map (cf. [7], Theorems 3.6 and $3.9)$.

(ii) For $\lambda \in \mathbb{R}$, let $\mu_{m}(\lambda)$ be defined as the unique $\mu \in \mathbb{R}$ such that the Dirichlet periodic problem $L u=\lambda m u+\mu_{m}(\lambda) u$ in $\Omega \times \mathbb{R}$ has a positive solution $u$. Then $\mu_{m}(\lambda)$ is well defined, $\mu_{m}(0)>0, \mu_{m}$ is concave and continuous, and a given $\lambda \in \mathbb{R}$ is a principal eigenvalue associated to the weight $m$ if and only if $\mu_{m}(\lambda)=0$ (cf. [7], Lemma 3.2). Also, if $\lambda_{1}(m)$ exists, then for $\lambda>0, \mu_{m}(\lambda)>0$ if and only if $\lambda<\lambda_{1}(m)$, and if $\lambda_{1}(m)$ does not exist, $\mu_{m}(\lambda)>0$ for all $\lambda>0$.

(iii) If $\mu_{m}(\lambda)>0$, then for all $h \in L_{T}^{r}, r>(N+2) / 2$, the problem

$$
\begin{cases}L u=\lambda m u+h & \text { in } \Omega \times \mathbb{R} \\ u=0 & \text { on } \partial \Omega \times \mathbb{R} \\ u T \text {-periodic } & \end{cases}
$$

has a unique solution $u \in W_{r, T}^{2,1}$ which is positive if $h>0$, and the solution operator $h \rightarrow u$ for this problem is continuous from $L_{T}^{r}$ into $C_{T}$ (cf. [8] Lemma 2.9). In particular, these conclusions apply for all $\lambda \geq 0$ if $\lambda_{1}(m)$ does not exists and for $0 \leq \lambda<\lambda_{1}(m)$ when $\lambda_{1}(m)$ exists.

Conversely, if (2.2) has a positive solution $h$ and $\lambda_{1}(m)$ exists, then $\lambda<$ $\lambda_{1}(m)$ (cf. [11], Remark $\left.2.1(\mathrm{e})\right)$. 
(iv) The following comparison principle holds: if $m_{1}, m_{2} \in L_{T}^{r}, P\left(m_{1}\right)>0$ and $m_{1} \leq m_{2}$ in $\Omega \times \mathbb{R}$, then $\lambda_{1}\left(m_{1}\right) \geq \lambda_{1}\left(m_{2}\right)$ and, if in addition $m_{1}<m_{2}$ in a set of positive measure, then $\lambda_{1}\left(m_{1}\right)>\lambda_{1}\left(m_{2}\right)$ (cf. [7], Remark 3.7).

Let

$$
P^{\circ}:=\text { interior of the positive cone of } C_{T}^{1+\theta,(1+\theta) / 2}
$$

The following lemma compiles some necessary facts about some semilinear periodic parabolic problems.

Lemma 2.2 Let $a, b \in L_{T}^{r}, r>N+2, a \geq 0$ and a not identically zero, and $0<p<1$. Consider the problem

$$
\begin{cases}L v=\lambda a v^{p}+b v & \text { in } \Omega \times \mathbb{R} \\ v=0 & \text { on } \partial \Omega \times \mathbb{R} \\ v T \text {-periodic } & \end{cases}
$$

(i) If $P(b)>0$, then for all $\lambda>0$, (2.3) has a solution $v=v_{\lambda} \in W_{r, T}^{2,1} \cap P^{\circ}$ if and only if $\lambda_{1}(b)>1$.

(ii) If $P(b) \leq 0$, then for all $\lambda>0$, (2.3) has a solution $v=v_{\lambda} \in W_{r, T}^{2,1} \cap P^{\circ}$. Moreover, in both cases the positive solution is unique and

$$
\begin{aligned}
\lim _{\lambda \rightarrow 0^{+}}\left\|v_{\lambda}\right\|_{L_{T}^{\infty}} & =0, \\
v_{\lambda} & =\lambda^{\frac{1}{1-p}} V, \text { with } V \in W_{r, T}^{2,1} \cap P^{\circ} \text { not depending on } \lambda
\end{aligned}
$$

Proof. Theorems 3.3 and 3.4 in [9] give some positive solution $v \in L_{T}^{\infty}$ in cases (i) and (ii) respectively. Since the operator $L^{-1}: L_{T}^{r} \rightarrow W_{r, T}^{2,1}$ is continuous (see e.g. [17], Section 4) it follows that $v \in W_{r, T}^{2,1}$, and from the Sobolev imbedding theorems (e.g. [16], Lemma 3.3, p. 80) and the strong maximum principle (e.g. [3], Theorem 13.5) we get that $v \in P^{\circ}$. Next, we note that $\xi \rightarrow\left(\lambda a \xi^{p}+b \xi\right) / \xi$ is decreasing, and thus the uniqueness assertion can be proved as in Theorem 3.5 in [10] (it can also be proved in a different way as in [11], Theorem 3.3). Observe also that, due to the homogeneity, (2.5) follows immediately from (2.3) taking $V$ as the positive solution of (2.3) with $\lambda=1$. To conclude the proof we note that (2.4) follows by uniqueness and letting $\lambda \rightarrow 0$ in (2.5).

We will need the following corollary of Young's inequality to prove the upper estimate for $\Lambda$ (for a proof, see [6], Lemma 3.6).

Lemma 2.3 Let $A, B \geq 0$ and $0<p<1<q$. Then for all $\xi \geq 0$ it holds that

$$
A \xi^{p}+B \xi^{q} \geq\left(\max \left\{\frac{q-1}{q-p}, \frac{1-p}{q-p}\right\}\right)^{-1} A^{\frac{q-1}{q-p}} B^{\frac{1-p}{q-p}} \xi:=c_{p, q} A^{\frac{q-1}{q-p}} B^{\frac{1-p}{q-p}} \xi
$$




\section{Main results}

Let

$$
\begin{aligned}
\delta(x) & :=\operatorname{dist}(x, \partial \Omega), \\
\Omega_{\varepsilon} & :=\{x \in \Omega: \delta(x) \leq \varepsilon\}
\end{aligned}
$$

Theorem 3.1 (i) Let $a, b \in L_{T}^{r}, r>N+2$, with $a \geq 0$ and a not identically zero. Then, there exists $\Lambda>0$ such that (1.3) has a solution $u=u_{\lambda} \in W_{r, T}^{2,1} \cap P^{\circ}$ for all $0<\lambda<\Lambda$, and $u_{\lambda}$ can be chosen such that

$$
\lim _{\lambda \rightarrow 0^{+}}\left\|u_{\lambda}\right\|_{L_{T}^{\infty}}=0
$$

Also, if $\lambda>\Lambda$ there is no positive solution for (1.3).

(ii) Assume in addition that $b \geq 0$. Then for all $0<\lambda<\Lambda$ there exists a minimal solution $u_{\lambda} \in W_{r, T}^{2,1} \cap P^{\circ}$.

Proof. Let $k>\left(1 / \lambda_{1}\left(b^{+}\right)\right)^{1 / q-1}$, where as usual we write $b=b^{+}-b^{-}$with $b^{+}=\max (b, 0)$ and $b^{-}=\max (-b, 0)$. Define $a_{k}:=k^{1-p} a, b_{k}:=k^{1-q} b$. Then, Lemma 2.2 (i) gives some $v=v_{\lambda} \in W_{r, T}^{2,1} \cap P^{\circ}$ solution of (2.3) with $a_{k}$ and $b_{k}^{+}$ in place of $a$ and $b$ respectively. Moreover, taking into account (2.4) we have that $L v \geq \lambda a_{k} v^{p}+b_{k} v^{q}$ for $\lambda>0$ small enough. In the same way, from Lemma 2.2 (ii) we get some $w=w_{\lambda} \in W_{r, T}^{2,1} \cap P^{\circ}$ solution of (2.3) with $a_{k}$ and $-b_{k}^{-}$in place of $a$ and $b$ respectively, and again from (2.4) we see that $L w \leq \lambda a_{k} w^{p}+b_{k} w^{q}$ for $\lambda>0$ small enough.

We claim that $v_{\lambda} \geq w_{\lambda}$. Indeed, note first that $w_{\lambda}$ is a subsolution of $(2.3)$ with $a_{k}$ and $b_{k}^{+}$in place of $a$ and $b$. On the other hand, since we can construct supersolutions $\widetilde{w}_{\lambda}$ of this last problem such that $\widetilde{w}_{\lambda} \geq c$ in $\Omega \times \mathbb{R}$ for all $c>0$ and all $\lambda>0$ (see [9], Lemma 2.3), Theorem 1 in [5] applies and thus we get a positive solution $u_{\lambda} \in L_{T}^{\infty}\left(w_{\lambda} \leq u_{\lambda} \leq \widetilde{w}_{\lambda}\right)$ of $(2.3)$ with $a_{k}$ and $b_{k}^{+}$in place of $a$ and $b$. Moreover, as in the proof of Lemma 2.1 we have $u_{\lambda} \in W_{r, T}^{2,1} \cap P^{\circ}$. Therefore, it follows that $v_{\lambda} \geq w_{\lambda}$ because if not we would have two solutions $u_{\lambda} \neq v_{\lambda}$, contradicting the uniqueness assertion in Lemma 2.1. Now, we can apply [5], Theorem 1, to obtain some $z=z_{\lambda} \in W_{r, T}^{2,1} \cap P^{\circ}\left(w_{\lambda} \leq z_{\lambda} \leq v_{\lambda}\right)$ solution of the Dirichlet periodic problem $L z=\lambda a_{k} z^{p}+b_{k} z^{q}$. It is easy to check that $u:=z / k$ is a solution for (1.3). Moreover, (3.1) holds since it is true for $v_{\lambda}$ and $0 \leq u_{\lambda} \leq v_{\lambda} / k$.

Let $\Lambda:=\sup \left\{\lambda>0\right.$ : there exists $u_{\lambda} \in W_{r, T}^{2,1} \cap P^{\circ}$ solution of $\left.(1.3)\right\}>0$. Let $0<\lambda<\Lambda$ and let $\lambda<\bar{\lambda}<\Lambda$ such that there exists $u_{\bar{\lambda}} \in W_{r, T}^{2,1} \cap P^{\circ}$ solution of (1.3) for $\lambda=\bar{\lambda}$. It follows that $u_{\bar{\lambda}}$ is a supersolution of (1.3). Moreover, as above we can choose $\underline{\lambda}>0$ small enough and $v_{\underline{\lambda}}>0$ solution of $L v_{\underline{\lambda}}=\underline{\lambda} a v_{\underline{\lambda}}^{p}-b^{-} v_{\underline{\lambda}}$ such that $v_{\underline{\lambda}}$ is a subsolution of (1.3) and $v_{\underline{\lambda}} \leq u_{\bar{\lambda}}$. Then, again Theorem 1 in [5] gives a solution of (1.3) and so the first part of the theorem follows. 
In order to prove (ii) we follow the ideas in [1]. Let $0<\lambda<\Lambda$, let $u$ be the solution of (1.3) found above, and let $v$ be the solution of (2.3) with $b \equiv 0$. Define a sequence $\left\{u_{j}\right\}_{j \in \mathbb{N}}$ such that

$$
L u_{j+1}=\lambda a u_{j}^{p}+b u_{j}^{q} \quad \text { in } \Omega \times \mathbb{R}
$$

where $u_{1}=v$. Since $b \geq 0$, it follows easily by induction and the maximum principle that $u_{j}$ is nondecreasing. Moreover, reasoning as in the second paragraph of the proof we get that $u_{1} \leq u$ and so in the same way as above we see that $u_{j} \leq u$ for all $j$. Let $u_{\infty}=\lim _{j \rightarrow \infty} u_{j}$. Then $u \leq u_{\infty} \in L_{T}^{\infty}$. Recalling that $L^{-1}: L_{T}^{r} \rightarrow C_{T}$ is compact (see e.g. [2], Section 5, also [8], Remarks 2.1 and 2.2 ), going to the limit in (3.2) we find that $u_{\infty}$ solves (1.3), and as in Lemma 2.2 $u_{\infty} \in W_{r, T}^{2,1} \cap P^{\circ}$ and this ends the proof.

Remark 3.2 Suppose $0 \leq a, b \in L_{T}^{r}$ such $b \leq c a$ for some positive constant $c$, and let $f(\xi):=\lambda a \xi^{p}+b \xi^{q}$. A computation shows that there exists some $\xi_{0}=\xi_{0}(a, b, p, q)$ such that $f^{\prime \prime}(\xi) \leq 0$ for all $0 \leq \xi \leq \xi_{0}$ a.e. in $\Omega \times \mathbb{R}$ and so $\xi \rightarrow f(\xi) / \xi$ is decreasing for such a $\xi$. Hence, as in Theorem 3.5 in [10] or in a similar way to Theorem 3.3 in [11] it can be proved that there exists at most one solution $u \in P^{\circ}$ of (1.3) such that $\|u\|_{L_{T}^{\infty}} \leq \xi_{0}$. This was proved (in a "variational manner") in [1], Theorem 2.2 for $L=-\Delta$ and $a=b \equiv 1$.

Theorem 3.3 Let $0 \leq a, b \in L_{T}^{r}, r>N+2$, with a not identically zero. Assume that

H1. Either there exist $\varepsilon, K>0$ and $\gamma>1-p-1 /(N+2)$ such that $a \leq K \delta^{\gamma}$ a.e. $(x, t) \in \Omega_{\varepsilon} \times \mathbb{R}$, or there exist $\varepsilon>0$ and $s>r$ such that $a \in L_{T}^{s}\left(\Omega_{\varepsilon} \times \mathbb{R}\right)$ and $p>1+1 / s-1 /(N+2)$, or $p>1+1 / r-1 /(N+2)$.

Then, there exists $\bar{\Lambda}>0$ such that a solution $u_{\lambda}$ of (1.3) can be chosen such that $\lambda \rightarrow u_{\lambda}$ is a $C^{1}$ increasing map from $(0, \bar{\Lambda})$ into $W_{r, T}^{2,1} \cap P^{\circ}$. Moreover, these $u_{\lambda}$ also satisfy (3.1).

Proof. Note first that if $u \in P^{\circ}$, any of the three conditions in H1 imply that $a / u^{1-p} \in L_{T}^{s}$ for some $s>N+2$. Indeed, suppose the first one is fullfilled. Then we can pick $\sigma>N+2$ such that $1-p<\gamma+1 / \sigma$. Since $u \geq c \delta$ for some $c>0$ we have

$$
\left\|a / u^{1-p}\right\|_{L^{\sigma}\left(\Omega_{\varepsilon} \times(0, T)\right)}^{\sigma} \leq c^{(p-1) \sigma} K^{\sigma} \int_{\Omega_{\varepsilon} \times(0, T)} \delta^{(\gamma+p-1) \sigma}<\infty
$$

the last inequality because $\sigma(\gamma+p-1)>-1$. So, $a / u^{1-p} \in L_{T}^{s}$ for $s:=$ $\min (\sigma, r)>N+2$. If the second or third condition hold, the proof is similar.

Let $\lambda>0, u_{\lambda}>0$ be the solution of (1.3) found in Theorem 3.1 (i), and let $m_{\lambda}:=\lambda p a u_{\lambda}^{p-1}+q b u_{\lambda}^{q-1}$. Then $m_{\lambda}$ belongs to $L_{T}^{s}$ for some $s>N+2$. We claim that the implicit function theorem can be applied in a point $\left(\lambda, u_{\lambda}\right)$ if $\lambda>0$ is small enough. Indeed, a direct computation shows that to in order to see this it 
suffices to prove that for a given $h \in L_{T}^{r}$ there is a unique solution $u \in W_{r, T}^{2,1}$ of the problem (2.2) with $m_{\lambda}$ in place of $m$ and that the solution operator for this problem is continuous. Thus, recalling Remark 2.1 (ii) and (iii), the claim will follow if $\lambda_{1}\left(m_{\lambda}\right)>1$ (if such $\lambda_{1}\left(m_{\lambda}\right)$ exists; if $\lambda_{1}\left(m_{\lambda}\right)$ does not exist we have nothing to prove). Now, reasoning as in the second paragraph of the proof of Theorem 3.1 (i) we get that (since $b \geq 0$ ) $u_{\lambda} \geq v_{\lambda}$, where $v_{\lambda}$ is the unique positive solution of (2.3) with $b \equiv 0$. Moreover, recalling (2.5) we have that $u_{\lambda} \geq \lambda^{1 /(1-p)} V$ with $V \in W_{r, T}^{2,1} \cap P^{\circ}$ satisfying $L V=a V^{p}$ and hence $1=\lambda_{1}\left(a V^{p-1}\right)$. So, taking into account (3.1) we get $m_{\lambda} \leq p a V^{p-1}+q b u_{\lambda}^{q-1} \rightarrow p a V^{p-1}$ in $L_{T}^{r}$ when $\lambda \rightarrow 0$, and then the last statement in Remark 2.1 (i) and the comparison principle in Remark 2.1 (iv) imply

$$
\lambda_{1}\left(m_{\lambda}\right)>\lambda_{1}\left(p a V^{p-1}+q b u_{\lambda}^{q-1}\right) \rightarrow\left(\lambda_{1}\left(a V^{p-1}\right)\right) / p=1 / p>1
$$

Thus the claim follows.

Let $(\underline{\Lambda}, \beta)$ be a maximal interval provided by the implicit function theorem in which $\lambda \rightarrow u_{\lambda}$ is a $C^{1}$ map into $W_{r, T}^{2,1} \cap P^{\circ}$. Differentiating (1.3) with respect to $\lambda$ we obtain

$$
\left(L-m_{\lambda}\right) \frac{\partial u}{\partial \lambda}=a u_{\lambda}^{p} \geq 0 \quad \text { in } \Omega \times \mathbb{R}
$$

If $P\left(m_{\lambda}\right) \leq 0$, then from Remark 2.2 (ii) and (iii) we have $\frac{\partial u}{\partial \lambda}>0$. On the other hand, if $\lambda_{1}\left(m_{\lambda}\right)$ exists, since $\lambda \rightarrow \lambda_{1}\left(m_{\lambda}\right)$ is continuous, again Remark 2.2 and (3.3) imply $\frac{\partial u}{\partial \lambda}>0$ for all $\lambda \in(\underline{\Lambda}, \bar{\Lambda}) \subset(\underline{\Lambda}, \beta)$. Therefore, $\lambda \rightarrow u_{\lambda}$ is increasing for such $\lambda$.

Suppose $\underline{\Lambda}>0$, and let $u_{j} \in P^{\circ}$ be the solutions of (1.3) corresponding to some sequence $\lambda_{j} \rightarrow \underline{\Lambda}$. A standard compactness argument gives some nonnegative $\underline{u} \in L_{T}^{\infty}$ solution of (1.3) for $\lambda=\underline{\Lambda}$. Moreover, since $u_{\lambda_{j}}$ are supersolutions of (2.3) with $b \equiv 0$, arguing as before and recalling (2.5) we find that $u_{\lambda_{j}} \geq \lambda_{j}^{1 /(1-p)} V$ for all $j$, where $L V=a V^{p}$. Hence, $\underline{u} \in P^{\circ}$. But then reasoning as above we can apply the implicit function theorem in the point $(\underline{\Lambda}, \underline{u})$, contradicting the maximality of $(\underline{\Lambda}, \beta)$. So, $\underline{\Lambda}=0$.

In order to prove the last statement of the theorem we proceed by contradiction. Suppose that (3.1) does not hold for these $u_{\lambda}$, and let $u_{0}=\lim _{\lambda \rightarrow 0^{+}} u_{\lambda}$. Then $u_{0} \geq 0$ and $u_{0}$ is not identically zero. Moreover, going to limit in (1.3) we obtain $L u_{0}=b u_{0}^{q}$ and thus

$$
\left\|u_{0}\right\|_{L_{T}^{\infty}} \geq 1 /\left(\left\|L^{-1}\right\|_{L_{T}^{r}, C_{T}}\|b\|_{L_{T}^{r}}\right)^{1 /(q-1)}:=\varepsilon_{0}
$$

On the other side, an inspection of the first part of the proof shows that the implicit function theorem can be applied in a point $\left(\lambda^{*}, u_{\lambda^{*}}\right)$ for any $\lambda^{*}$ small enough, where $u_{\lambda^{*}}$ are the solutions of (1.3) given by Theorem 3.1 (i) (and thus satisfying (3.1)). Therefore, we may assume that $\left\|u_{\lambda^{*}}\right\|_{L_{T}^{\infty}} \leq \varepsilon_{0} / 2$. But $\lambda \rightarrow u_{\lambda}$ is increasing, so $\left\|u_{0}\right\|_{L_{T}^{\infty}} \leq \varepsilon_{0} / 2$ which contradicts (3.4). 
Remark 3.4 Let us make some comments on condition H1. The only place in the proof where $\mathrm{H} 1$ is needed is to show that for $u \in P^{\circ}$ it holds that $a / u^{1-p} \in L_{T}^{r}$ for some $r>N+2$. Thus, if we had available linear theory for "singular" weights (as it is the case in the elliptic problem, see e.g. [12]), H1 could be removed.

Theorem 3.5 (i) Let $0 \leq a, b \in L_{T}^{r}, r>(N+2) / 2$, let $m:=a^{\frac{q-1}{q-p}} b^{\frac{1-p}{q-p}}$ and assume $P(m)>0$. Let $c_{p, q}$ be defined by (2.6). Then

$$
\Lambda \leq\left(\frac{\lambda_{1}(m)}{c_{p, q}}\right)^{\frac{q-p}{q-1}}:=\Lambda_{1}
$$

(ii) Let $a, b \in L_{T}^{r}, r>N+2$, with $a \geq 0$ and a not identically zero and $P(b)>0$. Then

$$
\Lambda \geq\left(\frac{(1-p)}{(q-p)\left\|L^{-1} B^{+}\right\|_{C_{T}, C_{T}}}\right)^{\frac{1-p}{q-1}} \frac{(q-1)}{(q-p)\left\|L^{-1}\right\|_{L_{T}^{r}, C_{T}}\|a\|_{L_{T}^{r}}}:=\Lambda_{2}
$$

where $B^{+}$denotes the operator multiplication by $b^{+}$.

Proof. Observe first that Hölder's inequality gives that $m \in L_{T}^{r}$. Let $\lambda>0, u>0$ be a solution for (1.3). Then, from (2.6) we see that $L u \geq c_{p, q} \lambda^{\frac{q-1}{q-p}} m u$ and so the last statement in Remark 2.1 (iii) gives $c_{p, q} \lambda^{\frac{q-1}{q-p}} \leq \lambda_{1}(m)$. Hence, $\lambda \leq \Lambda_{1}$ for all $\lambda>0$ such that there exists a positive solution for (1.3), and (3.5) follows.

Let us prove (ii). Note that it suffices to show that there exists a supersolution $u$ of (1.3) for $\lambda=\Lambda_{2}$. Indeed, this is enough because Lemma 2.4 in [9] gives subsolutions of this problem of the form $\varepsilon \Phi\left(\Phi \in P^{\circ}\right)$ for all $\varepsilon>0$ small enough and so, if such $u$ exists, from Theorem 1 in [5] we get a positive solution for (1.3). Moreover, given $b=b^{+}-b^{-}$, since any solution of (1.3) with $b^{+}$in place of $b$ is a supersolution of (1.3), reasoning as in the first part of the proof of Theorem 3.1 we may assume without loss of generality that $b \geq 0$.

Let $k=\left(\frac{q-p}{1-p}\left\|L^{-1} B\right\|\right)^{1 /(q-1)}$, and define $a_{k}:=k^{1-p} a, b_{k}:=k^{1-q} b$. We note that $\lambda_{1}\left(b_{k}\right)>1$. Indeed, since $\lambda_{1}(b)$ exists because $P(b)>0$, from (2.1) we have $1 \leq \lambda_{1}(b)\left\|L^{-1} B\right\|$ and thus

$$
\lambda_{1}\left(b_{k}\right) \geq \frac{q-p}{1-p} \lambda_{1}(b)\left\|L^{-1} B\right\| \geq \frac{q-p}{1-p}>1
$$

Then Lemma 2.1 (i) gives some positive solution $u$ of the Dirichlet periodic problem $L u=\Lambda_{2} a_{k} u^{p}+b_{k} u$ in $\Omega \times \mathbb{R}$. Clearly $u / k$ will be a supersolution of (1.3) for $\lambda=\Lambda_{2}$ if $\|u\|_{L_{T}^{\infty}} \leq 1$. Now, since $\left(L-b_{k}\right)^{-1}$ is a well defined continuous operator, after some computations we find

$$
\|u\|_{L_{T}^{\infty}}^{1-p} \leq \Lambda_{2}\left\|\left(L-b_{k}\right)^{-1}\right\|\left\|a_{k}\right\| \leq \frac{\Lambda_{2}\left\|L^{-1}\right\|\left\|a_{k}\right\|}{1-\left\|L^{-1} B_{k}\right\|}=1
$$

and this concludes the proof. 
Remark 3.6 The estimate in Theorem 3.3 (i) es essentially the one given (for the self-adjoint elliptic problem) in [6] (see Remark 2.12 and Corollary 2.7 there). We note that the proof given here is quite more simple. Note also that since $a, b \geq 0$, $P(m)>0$ reduces to $|\{a>0\} \cap\{b>0\}|>0$.

\section{References}

[1] A. AMBrosetti, H. BREZIS and G. CERAMI, Combined effects of concave and convex nonlinearities in some elliptic problems, J. Funct. Anal. 122 (1994), 519-543.

[2] D. DANERS, Domain perturbation for linear and nonlinear parabolic equations, J. Diff. Eqs. 129 (1996), 358-402.

[3] D. DANERS and P. KOCH-MEDINA, Abstract evolution equations, periodic problems and applications, Longman Research Notes 279, 1992.

[4] M. DELGADO and A. SUÁREZ, Positive solutions for the degenerate logistic indefinite superlinear problem: the slow diffusion case, Houston J. Math. 29 (2003), 801-820.

[5] J. DEUEL and P. HESS, Nonlinear parabolic boundary value problems with upper and lower solutions, Israel J. Math. 29 (1978), 92-104.

[6] D. G. DE FIGUEIREDO, J.-P. GOSSEZ and P. UBILLA, Local superlinearity and sublinearity for indefinite semilinear some elliptic problems, J. Funct. Anal. 199 (2003), 452-467.

[7] T. GODOY and U. KAUFMANN, On principal eigenvalues for periodic parabolic problems with optimal condition on the weight function, J. Math. Anal. Appl. 262 (2001), 208-220.

[8] T. GODOY and U. KAUFMANN, On positive solutions for some semilinear periodic parabolic eigenvalue problems, J. Math. Anal. Appl. 277 (2003), $164-179$.

[9] T. GODOY and U. KAUFMANN, On the existence of positive solutions for periodic parabolic sublinear problems, Abstr. Appl. Anal. 2003 (2003), 975-984.

[10] T. GODOY and U. KAUFMANN, Periodic parabolic problems with nonlinearities indefinite in sign, Publ. Mat. 51 (2007), 45-57.

[11] T. GODOY, U. KAUFMANN and S. PACZKA, Positive solutions for sublinear periodic parabolic problems, Nonlinear Anal. 55 (2003), 73-82. 
[12] J. HERNÁNDEZ, F. MANCEBO and J. VEGA, Positive solutions for singular nonlinear elliptic equations, Proc. Roy. Soc. Edinburgh Sect. A, to appear.

[13] P. HESS, Periodic-Parabolic Boundary Value Problems and Positivity, Longman Research Notes 247, 1992.

[14] P. HESS, M. POZIO and A. TESEI, Time periodic solutions for a class of degenerate parabolic problems, Houston J. Math. 21 (1995), 367-394.

[15] N. HIRANO and W. KIM, Multiple existence of periodic solutions for a nonlinear parabolic problem with singular nonlinearities, Nonlinear Anal. $\mathbf{5 4}$ (2003), 445-456.

[16] O. LADYSŽENKAJA, V. SOLONNIKOV and N. URAL'CEVA, Linear and quasilinear equations of parabolic type, Transl. Math. Mono 23, Amer. Math. Soc., 1968.

[17] G. LIEBERMAN, Time-periodic solutions of linear parabolic differential equations, Comm. Partial Diff. Eqs. 24 (1999), 631-664.

Received 27 May 2006; accepted 2 January 2007

Published Online First 21 September 2007

To access this journal online: http://www.birkhauser.ch 